\title{
THE SPASTICITY-RELATED QUALITY OF LIFE 6-DIMENSIONS INSTRUMENT IN UPPER-LIMB SPASTICITY: PART I DEVELOPMENT AND RESPONSIVENESS
}

Lynne TURNER-STOKES, DM, FRCP ${ }^{1,2}$, Klemens FHEODOROFF, MD ${ }^{3}$, Jorge JACINTO, MD ${ }^{4}$, Jeremy LAMBERT, PhD ${ }^{5}$, Christine DE LA LOGE, MSc ${ }^{5}$, Françoise CALVI-GRIES, MSc ${ }^{6}$, John WHALEN, BSc, MBA ${ }^{7}$, Andreas LYSANDROPOULOS, $\mathrm{MD}^{8}$, Pascal MAISONOBE, MSc ${ }^{6}$ and Stephen ASHFORD, PhD, FCSP ${ }^{1,2,9}$

From the ${ }^{1}$ Department of Palliative Care, Policy and Rehabilitation, Cicely Saunders Institute, Florence Nightingale Faculty of Nursing, Midwifery and Palliative Care, King's College London, London, UK, ${ }^{2}$ Regional Hyper-acute Rehabilitation Unit, Northwick Park Hospital, London, UK, ${ }^{3}$ Neurorehabilitation, Gailtal-Klinik, Hermagor, Austria, ${ }^{4}$ Centro de Medicina de Reabilitaçãode Alcoitão, Serviço de Reabilitação de adultos 3, Estoril, Portugal, ${ }^{5}$ Patient-Centred Outcomes, ICON plc, Lyon, France, ${ }^{6}$ Ipsen Pharma, Boulogne-Billancourt, France, ${ }^{7}$ Ipsen Biopharm Ltd, Slough, UK, ${ }^{8}$ Ipsen, Cambridge, MA, USA and ${ }^{9}$ Centre for Nursing, Midwifery and Allied Health Research, University College London Hospitals, London, UK

Objective: To describe the development of the Spasticity-related Quality of Life 6-Dimensions instrument (SQoL-6D) and its sensitivity to clinical change (responsiveness).

Design: Multicentre, prospective, longitudinal cohort study at 8 UK sites (NCT03442660).

Patients: Adults $(n=104)$ undergoing focal treatment of upper limb spasticity.

Methods: No condition-specific health-related quality of life tool is available for upper-limb spasticity of any aetiology. The SQOL-6D was developed to fulfil this need, designed to complement the Upper Limb Spasticity Index (which incorporates the Goal Attainment Scaling evaluation of upper limb spasticity [GASeous] tool) with targeted standardised measures. The 6 dimensions of the SQOL-6D (score range 0-4) map onto common treatment goal areas identified in upper-limb spasticity studies. A Total score (0-100) provides overall spasticity-related health status. To assess responsiveness, the SQOL-6D, Global Assessment of Benefit scale and "GASeous" were administered at enrolment and 8 weeks.

Results: Significant differences in mean SQOL-6D Total score change and effect sizes across patients rating "some benefit" $(0.51)$ and "great benefit" $(0.88)$ supported responsiveness.

Conclusion: The SQoL-6D is a promising new measure of health status in upper limb spasticity, that enables systematic assessment of the impact of this condition in relation to patients' priority treatment goals. A psychometric evaluation of SQoL-6D is presented separately.

Key words: quality of life; muscle spasticity; botulinum toxin; rehabilitation; surveys and questionnaires; health status.

Accepted Oct 7, 2021; Epub ahead of print Nov 2, 2021

J Rehabil Med 2021; 53: jrm002XX

Correspondence address: Lynne Turner-Stokes, Regional Rehabilitation Unit, Northwick Park Hospital, Watford Road, Harrow, Middlesex, HA1 3UJ, UK. E-mail: lynne.turner-stokes@doctors.org.uk

Q pasticity and spastic dystonia are common featu$\checkmark$ res of upper motor neurone syndrome following damage to the central nervous system (1). Both con-

\section{LAY ABSTRACT}

Upper-limb spasticity is a condition in which muscles become "tight", restricting use of the arm and hand. Questionnaires have been developed to assess a person's quality of life, but are not specifically designed to assess the impact of spasticity on a person. The Spasticity-related Quality of Life 6-Dimensions instrument (SQLL-6D) was created to address this deficiency. The development of the SQoL-6D is described here, along with analysis of its responsiveness to ensure that the SQoL-6D detects changes in spasticity symptoms following treatment (as reported by patients using other questionnaires). These analyses showed that the SQoL-6D captured changes in the burden of spasticity for people with this problem. Other technical psychometric properties of the SQoL-6D are reported in the companion paper available in this issue.

tribute to functional impairment, reduced activities of daily living and restricted social participation, which impact quality of life (QoL) and health status $(2,3)$. Upper-limb spasticity (ULS) typically occurs after a stroke or other acquired brain injury (4).

Botulinum toxin A (BoNT-A) injections are recommended for management of focal and regional spasticity $(5,6)$. However, while benefits are readily demonstrated at the level of impairment and of daily activities, understanding the impact of BoNT-A treatment on health-related QoL (HRQoL) is difficult as a result of the complex interaction of spasticity with other features of neurological disability, including motor weakness, contracture and limb deformity, cognitive, communicative, emotional and behavioural problems.

To date, attempts to show change in general aspects of HRQoL following treatment for spasticity using generic measures (Assessment of Quality of Life, EuroQoL-5 Dimensions-5 Levels (EQ-5D-5L), EuroQoL visual analogue scale, and Short-Form-36 (SF-36)) have largely been unsuccessful (7-10). This is partly because these instruments contain a range of items that are unlikely to be sensitive to focal intervention for localised spasticity, and partly because other neurological impairments and external factors are likely to impact more on these general instruments than spasticity itself. While generic measures 
have the advantage of enabling comparisons of disease burden and treatment benefit across diseases $(11,12)$, condition-specific measures of health have the potential advantage of being more responsive and clinically useful $(13,14)$. HRQoL measures specific to spasticity due to spinal cord injury do exist and are recommended for use alongside the SF-36 (15); however, no condition-specific tool is available for ULS of any aetiology.

The Spasticity-related Quality of Life 6-Dimensions instrument (SQoL-6D) was developed as a patientreported outcome tool to fulfil the need for a health status measure that would: $(i)$ be sensitive to the burden of patient experience in ULS and the changes following treatment, and (ii) might be used in the future for evaluation of treatments for ULS. The aim of this paper is to describe the development of the SQoL-6D and present results regarding its responsiveness to clinical change over time. The results of a formal psychometric evaluation of the SQoL-6D (validity and reliability), and consensus-based standards for the selection of health measurement instruments self-assessment are presented in a companion paper in this issue.

\section{METHODS}

\section{Context of SOoL-6D development}

Goal attainment scaling (GAS) is a process for setting patientcentred goals that can also be a sensitive measure of outcome from ULS treatment that enables identification of goals of importance to the patient and their carers (that are not otherwise identifiable using standardised measures) (16-18). The process also supports treatment planning and coordination of multidisciplinary patient-centred interventions (16-18).

Analysis of goal-setting statements from large ULS treatment studies and their classification using the World Health Organization (WHO) International Classification of Functioning, Disability and Health (ICF) (19) has identified 6 main goal areas for management of ULS in worldwide clinical practice. These are:

- Reduction of pain/discomfort;

- Control of spasms and other involuntary movements;

- Maintenance of upper limb movement range (i.e. avoiding contractures)

- Passive function (ease of caring for the affected limb, e.g. maintaining hygiene, skin integrity, dressing the limb);

- Active function (use of the affected limb, e.g. reaching, holding, grasping/releasing objects, lifting and carrying, or fine dexterity during activities such as meal preparation, household duties, work-related tasks, recreation/leisure activities);

- Mobility/balance (16, 17, 20, 21).

The Upper Limb International Spasticity observational study programme provided real-world evidence of the benefit of repeated cycles of BoNT-A treatment by first identifying GAS as the most widely applicable common treatment outcome measure, and then refining GAS tools together with standardised measures for use in clinical practice $(18,21-23)$.

Briefly, a structured approach to GAS was developed (GAS evaluation of ULS (GASeous) tool) to capture goal attainment for $1-3$ selected goals $(16,24,25)$. To record both patient- reported and clinician-rated elements of ULS, the ULS Index (ULSI) and Focal Spasticity Index (for upper- and lower-limb spasticity) $(26,27)$ were designed to measure:

- Severity of presentation and other confounders to recovery;

- Goals for treatment (using GASeous);

- Standardised measures of symptoms and function selected according to the patient's treatment goals (e.g. the $10-\mathrm{cm}$ visual analogue score for pain goals, Arm Activity measure (ArmA) for goals in passive or active function, etc.);

- Global benefit, as rated by both the patient and the treating clinician. However, these tools capture the diversity of presentation, goals and outcomes that reflect, but do not directly measure health status. In this context, the SQoL-6D was designed to complement the ULSI and GASeous (and be used in conjunction with them), by providing a systematic assessment of patients' experience of the main impacts of ULS, specifically in relation to a patient's priority treatment goals (Fig. 1). Patients with ULS have diverse presentations and widely differing goals for treatment (21). The SQoL-6D was developed on a formative model to capture health status across the range of patient experiences relating to spasticity.

\section{The SQoL-6D}

The SQoL-6D was developed following over a decade of research led by King's College London, including extensive analysis of goals and other outcomes from large international studies (16-18). The SQoL-6D is based on goal categories developed with patients and carers over a number of years to capture disease burden and concerns relevant to patients, and as such reflects recommendations by INVOLVE $(20,28)$. Further consultation with patient users was undertaken regarding question wording and presentation when delivered in paper and electronic versions.

The 6 dimensions (items) of the SQoL-6D map onto the same 6 goal areas identified above. It is important to note that, although the SQoL-6D is designed around common goal areas, each individual patient would usually only have treatment goals in 1-2 areas. While the whole SQoL-6D would provide an overall picture of spasticityrelated health status, when evaluating treatment response, it was anticipated that only dimensions relevant to the identified treatment goals would change. The SQoL-6D was therefore designed to be applied in a similarly targeted manner to the ULSI, so it is critical to understand the responsiveness of the SQol-6D, not only in terms of the overall score, but also at an individual item level.

Each dimension of the SQoL-6D uses a 5-level scale (score range $0-4)$ generally reflecting none (0), mild (1) moderate (2), severe (3), or extremely severe (4) problems. Higher scores therefore indicate a worse condition, mirroring the generic EQ-5D-5L item scores (29). The SQoL-6D Total score $(0-100)$ provides an overall picture of spasticity-related health status, and is computed as a linear transformation of the mean of the 6 dimension scores, with the direction of scoring inverted (higher scores indicating better health), in line with most HRQoL instruments.

Following refinement by an international advisory board of clinicians (see Acknowledgements) in minuted meetings, the SQoL-6D version 4.0 was accepted for further testing and use in July 2014.

The tool is freely available online, with user instructions, at https://www.kcl.ac.uk/cicelysaunders/resources/tools/thespasticity-related-quality-of-life-tool-(sqol-6d). The manual includes anchored information to help patients and clinicians identify the appropriate scoring level, for example:

- I have mild restriction in range of movement in my affected upper limb(s) (anchor: but I am able to stretch my joints out fully with assistance) 


\section{Why is SQoL-6D needed?}

In current clinical practice, no tool directly measures the health status of patients with upper limb spasticity (ULS)

\section{Current measures of treatment outcome}

\section{Six goal categories for Goal Attainment Scaling (GAS) in ULS ${ }^{1-3}$}

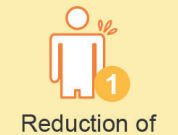

Reduction of
pain / discomfort

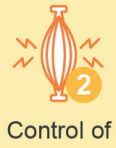

Control of
spasms

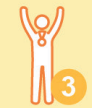

Maintenance of movement range

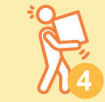

Active function (use of limb)

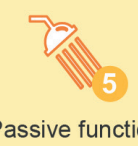

Passive function
(caring for limb)

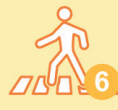

Mobility / balance

GAS evaluation of ULS (GASeous) is a structured approach to GAS specifically in ULS

that captures symptom presentation and goals. GASeous is incorporated into:

Focal Spasticity Index

Captures presentation

and goals in both ULS

and lower limb spasticity
ULS Index (ULSI)

A limited set of assessments that captures:

- Severity of presentation

- Confounders to recovery

- One to three treatment goals

\section{SQoL-6D maps onto the same six goal categories and complements the ULSI and GASeous}

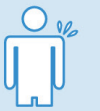<smiles>CC12C3C4CC5(C(C)(C)C)C3C1C5C42</smiles>

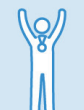<smiles>O=C1C2CC1C1CCC2C1</smiles><smiles>c1ccccc1</smiles>

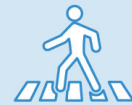<smiles>[O-][Co]</smiles>

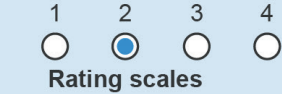

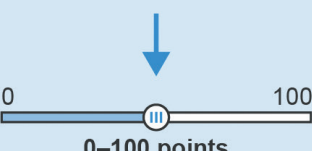

Each dimension of the SQOL-6D uses a five-level scale ( 0 to 4$)$ from 'no' to 'extremely severe' affliction in the goal area

Dimension scores are totalled and converted to the SQoL-6D Total score $(0$ to 100$)$. This provides an overall picture of spasticityrelated health status

SQoL-6D can facilitate economic evaluation of treatment and capture health status across the range of patient experiences relating to spasticity.

Fig. 1. Context and development of the spasticity-related quality of life 6-dimensions instrument (SQoL-6D). 1. Turner-Stokes et al. J Rehab Med 2010; 42: 81-89; 2. Ashford et al. Phy Res Int 2006; 11: 24-34; 3. Turner-Stokes et al. J Int Soc Phys Rehabil Med 2019; 2: 138-150.

- I have extremely severe restriction in range of movement in my affected upper limb(s) (anchor: so that I can barely move my affected limb at all)

\section{Study design}

To assess the psychometric properties of the SQoL-6D, including responsiveness as reported here, a multicentre, prospective, longitudinal, cohort study was conducted at $8 \mathrm{UK}$ sites (NCT03442660; see also the companion psychometric evaluation paper in this issue). Adults (aged $\geq 18$ years) receiving treatment for ULS as part of their routine clinical management were followed between May 2018 and October 2019. Enrolment was independent of therapeutic decisions, and required informed consent, an understanding of English and cognitive ability to understand the SQoL-6D questions, and fulfilment of protocol requirements, as judged by the investigator.

The SQoL-6D was self-administered at enrolment (Visit 1) and at 8 weeks ( \pm 2 weeks; Visit $2 /$ follow-up), by which time an intervention would be expected to impart its maximum effect on spasticity. The GASeous tool and the Global Assessment of Benefit Scale, a 5-point scale ( -2 much worse, -1 worse, 0 same, +1 some benefit, +2 great benefit) (21), were completed by both the investigator and the patient at Visit 2 .

\section{Intervention}

This study was a "non-clinical trial of investigational medicinal products" because it was set in the context of a cohort with clinical interventions for spasticity. Investigators were free to 
choose healthcare strategies in accordance with participant needs and routine clinical practice, but assessment tools were collected outside of normal clinical practice. Full details of interventions were not collected, as the performance of the SQoL-6D in real-life clinical practice was to be assessed regardless of intervention.

\section{Study population}

As responsiveness of the SQoL-6D (ability to detect change over time) was of key interest, calculation of sample size was based on this parameter. With an expected change in score of 5 points between baseline and follow-up, and a hypothesised standard deviation (SD) for the SQoL-6D Total score of 15 (effect size, 0.33), 87 patients were needed to detect a statistically significant change (paired $t$-test at $5 \%$; power of $80 \%$ ). Allowing for a drop-out rate of up to $13 \%$, the target for recruitment was set at 100 patients.

\section{Statistical analysis}

All analyses were pre-planned. The full analysis set (FAS; $n=98$ ) comprised all adults who completed Visit 1 . The "responsiveness" population $(n=90)$ comprised patients from the FAS who completed both Visits 1 and 2.

Responsiveness to change over time was assessed in the "responsiveness population" using analysis of variance (ANOVA) or $t$-tests at the $5 \%$ significance level, comparing change in scores from Visit 1-2 across groups defined by the level of "perceived benefit" (with the investigator- and patient-reported global assessment of benefit scale as "great benefit", "some benefit" and "no benefit" ("same", "worse" and "much worse" combined)) and by "overall goal attainment" (defined by GAS T-score $\geq 50$ ((i.e. goals achieved as expected or better) and GAS T-score $<50$ (goals not achieved as expected)).

We expected to see a positive relationship between improved health status (increase in total SQoL-6D) and global benefit, as perceived by both patients and clinicians. The sensitivity to change over time of the SQoL-6D individual dimension and Total scores between Visit 1 and 2 were also evaluated by the calculation of effect sizes (mean difference divided by the pooled SD at baseline). An effect size of 0.8 or more represents a "large", 0.5 a "medium" and 0.2 a "small" change (30).

Electronic data capture was used whenever possible, which did not allow patients to skip items or save incomplete entries, to minimise missing data; no imputations were made for missing data. Statistical Analysis System (SAS®) version 9.4 (SAS Institute Inc., Cary, NC, USA) was used for statistical analysis.

\section{RESULTS}

\section{Patient disposition and socio-demographics}

Across the 8 study sites, 104 patients were enrolled (Fig. 2; 3-22 patients per site). Demographic and disease characteristics are shown in Table I. More detailed demographic and disease characteristics are provided in the companion psychometric evaluation paper in this issue. This was a relatively young population (mean age 53 years) with chronic spasticity (median duration 5.4 (range $0-38.5$ ) years). Overall, the study population demographics were comparable to those of other published large international cohorts with $\operatorname{ULS}(18,21)$.

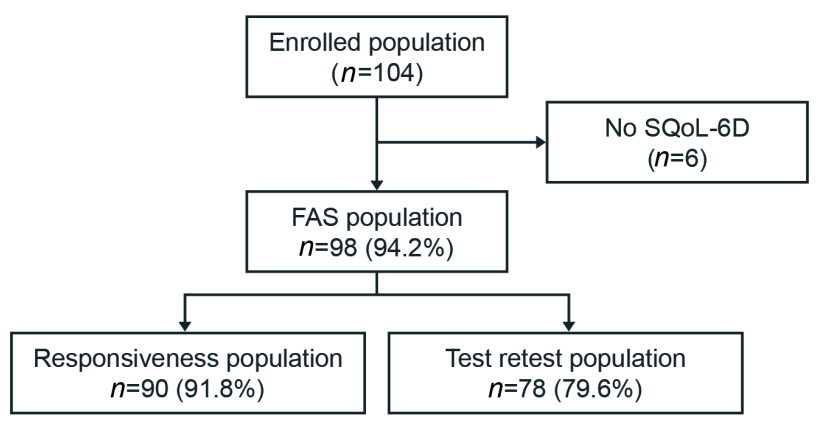

Fig. 2. Patient disposition. Percentages were calculated based on the enrolled population for the full analysis set (FAS) population, and based on the FAS population for the responsiveness population. SQoL-6D: Spasticity Quality of Life-6 Dimensions instrument.

\section{Responsiveness to clinical changes}

The mean change in the SQoL-6D Total score between enrolment and follow-up was 11.9 (95\% CI 9.4, $14.4)$, with reduction in mean item scores ranging from -0.3 to -0.6 (Table II). ANOVA demonstrated statistically significant differences in the change in SQoL-6D Total score between patients who reported "some benefit" compared with "great benefit" ( $p=0.0008$; Table III) and similar differences were seen between groups defined by investigator-reported benefit $(p=0.0014)$

These results were further supported by effect sizes, which indicated a large change for the patients reporting "great benefit" (effect size $=0.98$ ); a medium change for those reporting "some benefit" (effect size $=0.52)$; and no change for those reporting "no benefit" (effect size $=-0.04$ ).

Responsiveness by item showed a logical pattern

Table I. Patient demographic and disease characteristics

\begin{tabular}{ll}
\hline Characteristics & $\begin{array}{l}\text { FAS } \\
(n=98)\end{array}$ \\
\hline Age, years, mean (95\% CI) [Range] & $53.0(49.9,56.0)[19-82]$ \\
Male, $n(\%)$ & $65(66.3)$ \\
Ethnic group, $n(\%)$ & $85(86.7)$ \\
White & $11(11.2)$ \\
Asian-Asian British & $2(2.0)$ \\
Other & \\
Duration since ULS onset, months & $92.7(73.5,111.8)$ \\
Mean (95\% CI) & $65.0(22.4,123.8)[0-462]$ \\
Median, IQR, [Range] & \\
Arm affected ${ }^{\mathrm{a}}, n$ (\%) & $52(53.1)$ \\
Left & $46(46.9)$ \\
Right & \\
Affected upper limb, $n$ (\%) & $45(45.9)$ \\
Dominant & $48(49.0)$ \\
Non-dominant & $5(5.1)$ \\
Both arms & \\
\hline
\end{tabular}

Percentages are based on the number of patients with available data in the FAS population. ${ }^{\text {If }}$ both arms were affected, the most severely affected arm was studied; if both arms were affected with the same severity, data for right arm was studied.95\% CI: 95\% confidence interval; FAS: full analysis set; IQR: interquartile range; SD: standard deviation; ULS: upper limb spasticity. 
Table II. Mean scores at enrolment and follow-up and change scores

\begin{tabular}{|c|c|c|c|c|c|}
\hline SQoL-6D dimension & & Enrolment $(n=98)$ & Follow-up $(n=90)$ & Change score $(n=90)$ & $p$-value \\
\hline \multirow[t]{2}{*}{ 1. Pain/discomfort } & Mean (SD) & $1.8(1.2)$ & $1.3(1.1)$ & $-0.5(1.1)$ & $<0.0001$ \\
\hline & $95 \% \mathrm{CI}$ & $(1.5,2.0)$ & $(1.1,1.5)$ & $(-0.7,-0.3)$ & \\
\hline 2. Involuntary movements or spasms & Mean (SD) & $1.6(1.3)$ & $1.1(1.1)$ & $-0.6(1.1)$ & $<0.0001$ \\
\hline \multirow[t]{2}{*}{ 3. Restricted range of movement } & Mean (SD) & $2.7(1.0)$ & $2.1(1.0)$ & $-0.6(1.1)$ & $<0.0001$ \\
\hline & $95 \%$ CI & $(2.5,2.9)$ & $(1.9,2.4)$ & $(-0.8,-0.4)$ & \\
\hline \multirow[t]{2}{*}{ 4. Caring for the affected limb } & Mean (SD) & $2.1(1.3)$ & $1.7(1.4)$ & $-0.4(0.9)$ & $<0.0001$ \\
\hline & $95 \%$ CI & $(1.8,2.3)$ & $(1.4,2.0)$ & $(-0.6,-0.2)$ & \\
\hline \multirow[t]{2}{*}{ 5. Using the affected limb } & Mean (SD) & $3.4(0.7)$ & $3.1(0.9)$ & $-0.3(0.8)$ & 0.0005 \\
\hline & $95 \%$ CI & $(3.3,3.6)$ & $(2.9,3.3)$ & $(-0.5,-0.1)$ & \\
\hline \multirow[t]{2}{*}{ 6. Mobility/balance } & Mean (SD) & $2.4(1.2)$ & $2.0(1.4)$ & $-0.5(1.1)$ & $<0.0001$ \\
\hline & $95 \% \mathrm{CI}$ & $(2.2,2.7)$ & $(1.7,2.3)$ & $(-0.7,-0.3)$ & \\
\hline \multirow[t]{3}{*}{ Total score } & Mean (SD) & $41.4(18.8)$ & $53.1(20.3)$ & 11.9 (11.9) & \\
\hline & $95 \%$ CI & $(37.6,45.1)$ & $(48.8,57.4)$ & $(9.4,14.4)$ & $<0.0001$ \\
\hline & [Range] & {$[0.0-79.2]$} & {$[8.3-95.8]$} & {$[-12.5-37.5]$} & \\
\hline
\end{tabular}

SQoL-6D individual dimension scores range from 0 to 4 , with higher scores indicating worse outcome. SQoL-6D Total score ranges from 0 to 100 , with higher scores indicating better quality of life. $95 \%$ CI: 95\% confidence interval; SQoL-6D: spasticity-related quality of life 6-dimensions instrument.

(although not reaching statistical significance) of larger improvements in SQoL-6D scores for patients with greater benefit, as rated by the clinician, except for pain/discomfort (Fig. 3). Similarly, when using the patient Global Assessment of Benefit scale, dimension and Total scores indicated greater mean improvements for patients with greater benefits, except for item 5 "using the affected limb". Comparison of subgroups identified $p$-values of $<0.01$ for item 6 "mobility/balance" and for the SQoL-6D Total score.

Trends were generally as expected, with patients having reached their GASeous goals showing larger mean improvements than those who did not. However, none of the between-group differences reached statistical significance, except for mobility/balance $(p=0.022)$. Patients not reaching their goals presented an effect size of 0.37 , suggesting a small improvement observed in these patients, while those achieving their goals presented an improvement of medium to large size $($ effect size $=0.68)$.

\section{DISCUSSION}

The SQoL-6D is the first condition-specific self-reported questionnaire developed for adult patients with ULS of any aetiology, to assess change in health status. The SQoL-6D is designed to be simple and easy to selfadminister, and maps onto the 6 key goal areas that have been identified previously in worldwide clinical practice as relevant to patients with ULS and their carers.

Here, the SQoL-6D demonstrated responsiveness to clinical change in a UK multicentre cohort of patients with ULS. The limited level of association between the SQoL-6D Total score and GAS T-score was not unduly surprising, as most patients have only 1-2 stated goals, which are therefore by definition restricted to 1-2 items of the SQoL-6D. As noted earlier, the SQoL-6D was originally designed to be applied in a targeted manner, selecting only the items relevant to the patient's chosen goal areas to measure interventionrelated change. However, having a global tool with a Total score provides a standardised tool that supports

Table III. Change in spasticity-related quality of life 6-dimensions instrument (SQoL-6D) Total score between enrolment and follow-up by subgroups

\begin{tabular}{|c|c|c|c|c|}
\hline \multirow[b]{2}{*}{ Subgroups of clinical benefit } & \multicolumn{4}{|c|}{ SQoL-6D Total Score } \\
\hline & $n$ & Mean $(95 \% \mathrm{CI})$ & $p$-value & Effect size \\
\hline \multicolumn{5}{|c|}{ Investigator Global Assessment of Benefit scale } \\
\hline Some benefit & 56 & $10.1(7.1,13.1)$ & $p=0.0008$ & 0.52 \\
\hline Great benefit & 29 & $17.5(13.3,21.8)$ & & 0.98 \\
\hline \multicolumn{5}{|c|}{ Patient Global Assessment of Benefit scale ${ }^{a}$} \\
\hline Same/worse/much worse & 5 & $2.5(-10.5,15.5)$ & & 0.19 \\
\hline Some benefit & 51 & $9.5(6.6,12.4)$ & $p=0.0014$ & 0.51 \\
\hline Great benefit & 31 & $17.6(13.1,22.1)$ & & 0.88 \\
\hline Missing $^{a}$ & 3 & & & \\
\hline \multicolumn{5}{|l|}{ GAS T-score at follow-up } \\
\hline GAS T-score $<50$ & 20 & $7.9(1.5,14.3)$ & $p=0.0925$ & 0.37 \\
\hline GAS T-score $\geq 50$ & 69 & $13.0(10.3,15.8)$ & & 0.68 \\
\hline Missing $^{a}$ & 1 & & & \\
\hline
\end{tabular}

${ }^{a}$ There were few missing cases; no specific measures were taken to account for missing data. Data are derived from the responsiveness population ( $n=90$ ). Analysis of variance and $t$-tests were used to derive p-values for global assessment of benefit scales, and GAS T-scores, respectively. $95 \%$ CI: $95 \%$ confidence interval; GAS: goal attainment scaling; SQoL-6D: spasticity-related quality of life 6-dimensions instrument. 
p. 6 of 8 L. Turner-Stokes et al.

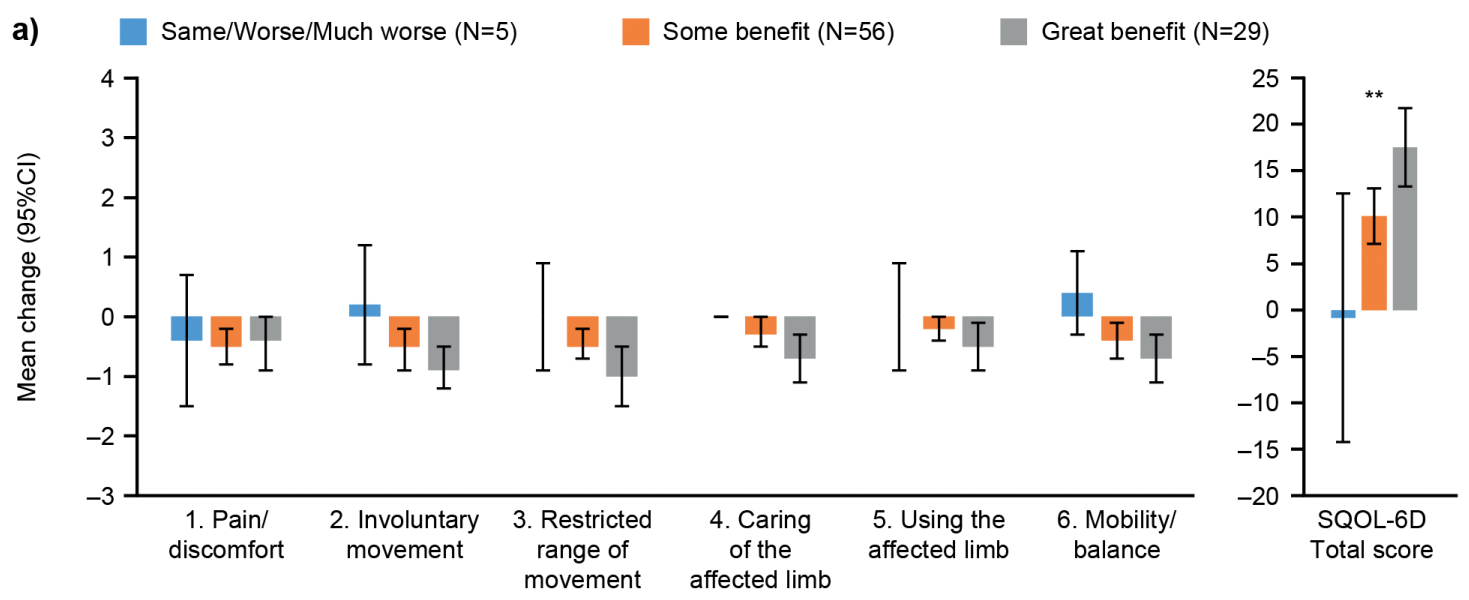

b) Same/Worse/Much worse $(\mathrm{N}=5) \quad$ Some benefit $(\mathrm{N}=51) \quad$ Great benefit $(\mathrm{N}=31)$

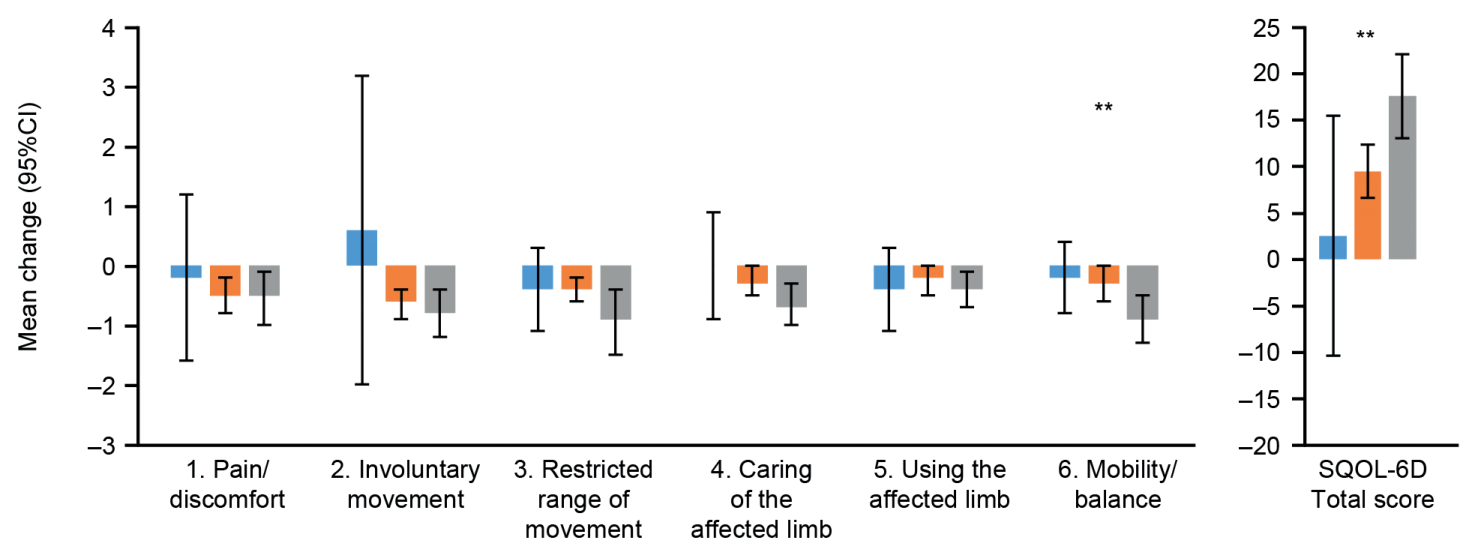

c) GAS-T score $<50(\mathrm{~N}=20)$

GAS-T score $\geq 50(\mathrm{~N}=69)$
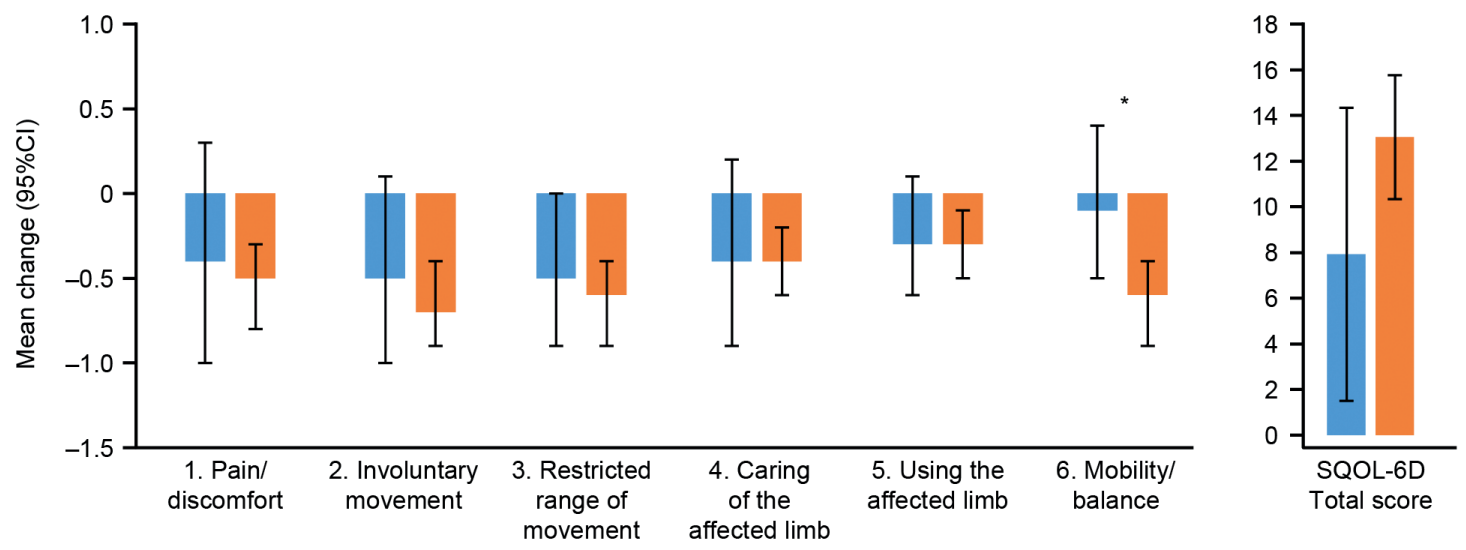

Fig. 3. Mean ( $95 \% \mathrm{CI}$ ) change in Spasticity Quality of Life-6 Dimensions instrument (SQoL-6D) scores by subgroups at follow-up. (a) Clinician Global Assessment of Benefit scale. (b) Patient Global Assessment of Benefit scale. (c) GAS T-scores. Data are derived from the Responsiveness Population $(n=90) . * p<0.05 * * p<0.01$. Analysis of variance (ANOVA) was used for the Total score, Kruskal-Wallis for dimension scores and $t$-tests for GAS T-scores. Note: Parametric and non-parametric tests led to similar $p$-values. $95 \%$ CI: $95 \%$ confidence interval; GAS: goal attainment scaling .

pooled analysis and group comparison. Hence, we first explored the performance of the overall tool (and the study was powered with that in mind). A larger sample would be necessary with representation across all goal areas to explore more targeted application of the tool.

Similarly, although a larger sample would be needed to define a responder threshold for the SQoL-6D Total score, these preliminary data allow us to provide 
some guidance for interpreting within-patient score change over time. Using anchor-based methods, the mean change in SQoL-6D score for patients who have reported "some benefit" on the Global Assessment of Benefit scale was 9.5, and 10.1 when using the clinician Global Assessment of Benefit scale; therefore, a Total score change of about 10 points could be considered to represent a meaningful improvement. It should also be highlighted that distribution-based methods indicate that a Total score change of 10 points represents more than half the baseline SD of 18.8, which is generally considered as benchmark to define effects of medium size. Comparison of results from patients who improved following treatment vs those who did not was not possible using the receiver operating characteristic curve, as there was an insufficient number of patients ( $n=5)$ who reported "no benefit" on the Global Assessment of Benefit scale; further research is therefore required to define a minimally important difference.

The authors acknowledge a number of strengths and weaknesses to this study. Strengths include the multicentre sample, and the fact that it was conducted in real-life clinical practice, both of which help to support the generalisability of the findings. Although the sample size of just under 100 patients was fairly modest, the study was adequately powered for its intended purpose. Weaknesses include the fact that the study was confined to one country. Further testing should be conducted in a larger sample allowing analysis of individual items in patients targeting the corresponding therapeutic goals.

In conclusion, the SQoL-6D was developed to fulfil the need for a health-status measure specific for adults with ULS of any aetiology. Results of a multicentre study show the SQoL-6D is responsive to detecting clinical change in patients of differing clinical outcome following focal treatment of ULS. A companion paper in this issue provides further technical results related to the psychometric evaluation of the SQoL-6D reliability and validity.

\section{ACKNOWLEDGEMENTS}

Members of the international advisory board (speciality) who refined the SQoL-6D were: Stephen Ashford (Neurological Rehabilitation, UK); Klemens Fheodoroff(Neurorehabilitation, Austria); Jorge Jacinto (Physical Medicine and Rehabilitation, Portugal); Ian Baguley (Neurological Rehabilitation, Australia); Melissa Knott (Neurological Rehabilitation, Australia); and Stephen De Graaff (Rehabilitation and Pain, Australia). The authors also thank Nicola Winstone, DPhil, and Germanicus Hansa-Wilkinson, MSc, of Ashfield MedComms, UK, an Ashfield Health company, for providing medical writing and editorial support, which was sponsored by Ipsen in accordance with Good Publication Practice guidelines.

Disclosures. LTS has a specific interest in outcomes evaluation and has published on the use of Goal Attainment Scaling in this context, as well as standardised measures, such as the GASeous, NIS, Arm and Leg activity measures (ArmA and LegA). These tools are freely available. She has received honoraria and travel grants from Ipsen and Merz. She has no personal financial interest in any of the material mentioned in this article. KF has a specific interest in ICF-based outcomes evaluation goal setting. $\mathrm{He}$ has received unrestricted research grants from Ipsen and has received honoraria from Allergan (now AbbVie), Ipsen and Merz. He has no personal financial interest in any of the material mentioned in this article. JJ has received honoraria for services such as scientific advisor, clinical researcher, trainer and speaker from Allergan (now AbbVie), Ipsen and Merz. JL is an ICON plc employee and a paid consultant to Ipsen. CDLL is a paid consultant to ICON plc. FCG, JW, AL and PM are employees of Ipsen. SA has a specific interest in outcomes evaluation and has published on the use of Goal Attainment Scaling in this context, as well as standardised measures such as the Arm and Leg activity measures (ArmA and LegA). These tools are freely available. He has received 2 unrestricted research grants from Ipsen and has received honoraria from Allergan (now AbbVie), Ipsen, Merz and Nutricia. He has no personal financial interest in any of the material mentioned in this article.

Data sharing. Ipsen will share aggregated data that underlie the results reported in this article with qualified researchers who provide a valid research question, and subject to an appropriate data sharing agreement. Study documents, such as the study protocol and clinical study report, are not always available. Proposals should be submitted to DataSharing@Ipsen.com and will be assessed by a scientific review board. Data are available beginning 6 months and ending 5 years after publication; after this time, only raw data may be available.

Ethics. This study was an interventional, non-clinical trial of an investigational medicinal product (non-CTIMP) and therefore fell outside the scope of the European Union (EU) Directive 2001/20/EC and the EU Directive 2005/28/EC. The study was defined as "interventional", not because of the use of an investigational product, but because of data collection procedures imposing multiple questionnaire completion and one on-site visit that were not necessarily part of routine clinical practice. Before initiating the study, ethics permission was granted by the UK Health Research Authority IRAS number: 232862.

All patients provided written informed consent prior to participating in any study-related activities.

Funding and management. This study was sponsored by Ipsen. Statistical analysis was performed by an external CRO (ICON plc) on behalf of Ipsen Pharma. London North West University Healthcare Trust (Northwick Park Hospital), UK, and King's College London, UK, contributed to time spent by the lead author in preparation of the manuscript, and the last author (SA) in supporting approvals and oversite of data collection.

\section{REFERENCES}

1. Pandyan AD, Gregoric M, Barnes MP, Wood D, Van Wijck F, Burridge J, et al. Spasticity: clinical perceptions, neurological realities and meaningful measurement. Disabil Rehabil 2005; 27: 2-6.

2. Barnes M, Kocer S, Murie Fernandez M, Balcaitiene J, Fheodoroff $\mathrm{K}$. An international survey of patients living with spasticity. Disabil Rehabil 2017; 39: 1428-1434.

3. Ghai A, Garg N, Hooda S, Gupta T. Spasticity - pathogenesis, prevention and treatment strategies. Saudi J Anaesth 2013; 7: 453-460.

4. Angulo-Parker FJ, Adkinson JM. Common etiologies of upper extremity spasticity. Hand Clin 2018; 34: 437-443.

5. Esquenazi A, Novak I, Sheean G, Singer BJ, Ward AB. International consensus statement for the use of botulinum toxin treatment in adults and children with neurological impair- 
ments - introduction. Eur J Neurol 2010; 17 (Suppl 2): 1-8.

6. Royal College of Physicians. Spasticity in adults: Management using botulinum toxin. National Guidelines. 2nd edition. 2018. Available from: https://www.rcplondon. ac.uk/guidelines-policy/spasticity-adults-managementusing-botulinum-toxin [Accessed 2021 Oct 18].

7. McCrory P, Turner-Stokes L, Baguley IJ, De Graaff S, Katrak $P$, Sandanam J, et al. Botulinum toxin A for treatment of upper limb spasticity following stroke: a multi-centre randomized placebo-controlled study of the effects on quality of life and other person-centred outcomes. J Rehabil Med 2009; 41: 536-544.

8. Childers MK, Brashear A, Jozefczyk P, Reding M, Alexander $D$, Good D, et al. Dose-dependent response to intramuscular botulinum toxin type A for upper-limb spasticity in patients after a stroke. Arch Phys Med Rehabil 2004; 85: 1063-1069.

9. Cuenca Zaldívar JN, Calvo S, Bravo-Esteban E, Oliva Ruiz P, Santi-Cano MJ, Herrero P. Effectiveness of dry needling for upper extremity spasticity, quality of life and function in subacute phase stroke patients. Acupunct Med 2020: 964528420947426.

10. Roncoroni LP, Weiss D, Hieber L, Sturm J, Börtlein A, Mayr I, et al. Health-related quality of life outcomes from botulinum toxin treatment in spasticity. Toxins (Basel) 2020; 12: 292.

11. Contopoulos-Ioannidis DG, Karvouni A, Kouri I, Ioannidis JP. Reporting and interpretation of SF-36 outcomes in randomised trials: systematic review. BMJ 2009; 338: a3006.

12. FrendI DM, Ware JE, Jr. Patient-reported functional health and well-being outcomes with drug therapy: a systematic review of randomized trials using the SF-36 health survey. Med Care 2014; 52: 439-445.

13. Wiebe S, Guyatt G, Weaver B, Matijevic S, Sidwell C. Comparative responsiveness of generic and specific qualityof-life instruments. J Clin Epidemiol 2003; 56: 52-60.

14. Wilson IB, Cleary PD. Linking clinical variables with healthrelated quality of life. A conceptual model of patient outcomes. JAMA 1995; 273: 59-65.

15. Ertzgaard P, Nene A, Kiekens C, Burns AS. A review and evaluation of patient-reported outcome measures for spasticity in persons with spinal cord damage: Recommendations from the Ability Network - an international initiative. J Spinal Cord Med 2020; 43: 813-823.

16. Turner-Stokes L, Baguley I, De Graff S, McCrory P, Katrak P, Davies $L$, et al. Goal attainment scaling in the evaluation of treatment of upper limb spasticity with botulinum toxin: a secondary analysis from a double blind placebo controlled randomised clinical trial. J Rehabil Med 2010; 42: 81-89.

17. Ashford S, Turner-Stokes L. Goal attainment for spasticity management using botulinum toxin. Physiother Res Int 2006; 11: 24-34.

18. Turner-Stokes L, Fheodoroff K, Jacinto J, Maisonobe P, Ashford S. ULIS (Upper Limb International Spasticity), a 10-year odyssey: An international, multicentric, longitudinal cohort of person-centered spasticity management in real-life practice. J Int Soc Phys Rehabil Med 2019; 2:
138-150.

19. World Health Organisation. International Classification of Functioning, Disability and Health (ICF). 2001. Available from: https://www.who.int/standards/classifications/ international-classification-of-functioning-disability-andhealth [Accessed 2021 Oct 26].

20. Ashford S, Fheodoroff K, Jacinto J, Turner-Stokes L. Common goal areas in the treatment of upper limb spasticity: a multicentre analysis. Clin Rehabil 2016; 30: 617-622.

21. Turner-Stokes L, Fheodoroff K, Jacinto J, Maisonobe P. Results from the Upper Limb International Spasticity Study-II (ULIS-II): a large, international, prospective cohort study investigating practice and goal attainment following treatment with botulinum toxin $A$ in real-life clinical management. BMJ Open 2013; 3: e002771.

22. Turner-Stokes L, Fheodoroff K, Jacinto J, Maisonobe $P$, Zakine B. Upper limb international spasticity study: rationale and protocol for a large, international, multicentre prospective cohort study investigating management and goal attainment following treatment with botulinum toxin A in real-life clinical practice. BMJ Open 2013; 3: e002230.

23. Turner-Stokes L, Jacinto J, Fheodoroff $K$, Brashear A, Maisonobe $\mathrm{P}$, Lysandropoulos $\mathrm{A}$, et al. Longitudinal goal attainment with integrated upper limb spasticity management including repeat injections of botulinum toxin $A$ : Findings from the prospective, observational Upper Limb International Spasticity (ULIS-III) cohort study. J Rehabil Med 2021; 53: jrm00157.

24. Turner-Stokes L, Ashford S, Jacinto J, Maisonobe P, Balcaitiene J, Fheodoroff K. Impact of integrated upper limb spasticity management including botulinum toxin $A$ on patient-centred goal attainment: rationale and protocol for an international prospective, longitudinal cohort study (ULIS-III). BMJ Open 2016; 6: e011157.

25. Turner-Stokes L, Ashford SA. The GAS-eous tool. 2013. Available from: http://www.kcl.ac.uk/lsm/research/ divisions/cicelysaunders/attachments/Tools-GASeousGASeous-tool.pdf [Accessed 2021 Oct 18].

26. Ashford S, Turner-Stokes L. The Focal Spasticity Index. 2018; Available from: https://www.kcl.ac.uk/cicelysaunders/resources/tools/focal-spasticity-index-sa-2018.pdf [Accessed 2021 Oct 18].

27. Ashford S, Turner-Stokes L. The Upper Limb Spasticity Index. 2014; Available from: https://www.kcl.ac.uk/cicelysaunders/resources/tools/the-upper-limb-spasticityindex-13.pdf [Accessed 2021 Oct 18].

28. The UK Public Involvement Standards Development Partnership. The UK Standards for Public Involvement. Available from: https://sites.google.com/nihr.ac.uk/pistandards/home [Accessed 2021 Oct 18].

29. Herdman M, Gudex C, Lloyd A, Janssen M, Kind P, Parkin $D$, et al. Development and preliminary testing of the new five-level version of EQ-5D (EQ-5D-5L). Qual Life Res 2011; 20: 1727-1736.

30. Cohen J. Statistical power analysis for the behavioral sciences: New York: Academic Press; 1969. 